\title{
Wiener Astronomen und Kleinplaneten
}

\author{
Anneliese Schnell \\ Institut für Astronomie, Türkenschanzstrasse 17, 1180 Vienna, Austria
}

\begin{abstract}
Mainly in the second half of the 19th century and at the beginning of the 20th century Viennese astronomers worked on Minor Planets, their discovery, the determination of positions and orbits. Most important was Johann Palisa. In cooperation with Max Wolf from Heidelberg he published the very first photographic stellar charts.
\end{abstract}

Bis zum Beginn des Spacewatch Projekts 1980 lag die Wiener Sternwarte bei einer Reihung der Entdeckung von Asteroiden mit 97 Objekten unter den 20 führenden Instituten der Welt. Diese herausragende Position verdankt sie vor allem einem Astronomen, Johann Palisa. Palisa (1848 - 1925) entdeckte während seines Lebens 121 Asteroiden, davon 28 in der Zeit, in der er Direktor der Sternwarte Pola war.

Schon die Astronomen an der alten Wiener Sternwarte beschäftigten sich mit diesen Objekten. Bereits in der ersten, 1835 erschienenen Auflage von J. J. Littrows Lehrbuch "Die Wunder des Himmels" (Littrow 1835) werden die damals bekannten vier Asteroiden erwähnt und es wird die Vermutung geäußert, dass es noch mehr dieser Objekte gibt. Ab 1831 gaben die Direktoren der Wiener Sternwarte den sogenannten "Kalender für alle Stände" heraus, der ab 1852 regelmäßig einen Beitrag über neu entdeckte Asteroiden enthält; in ihm wurden Untersuchungen über Bahnelemente und Bahnen veröffentlicht. Im Kalender findet man Berichte über die internationale Zusammenarbeit auf diesem Gebiet, über die Aufteilung von Beobachtungen und Bahnbestimmungen auf mehrere Sternwarten, in Wien wurden wie in Berlin und Kremsmünster alle mit dem vorhandenen Instrumentarium erreichbaren Objekte beobachtet sowie Bahnbestimmungen durchgeführt (z.B. Calliope, Lätitia, Leda).

Die Astronomen der alten Wiener Sternwarte beschäftigten sich also mit den ihnen zur Verfügung stehenden Mitteln ausführlich mit Asteroiden. Für 

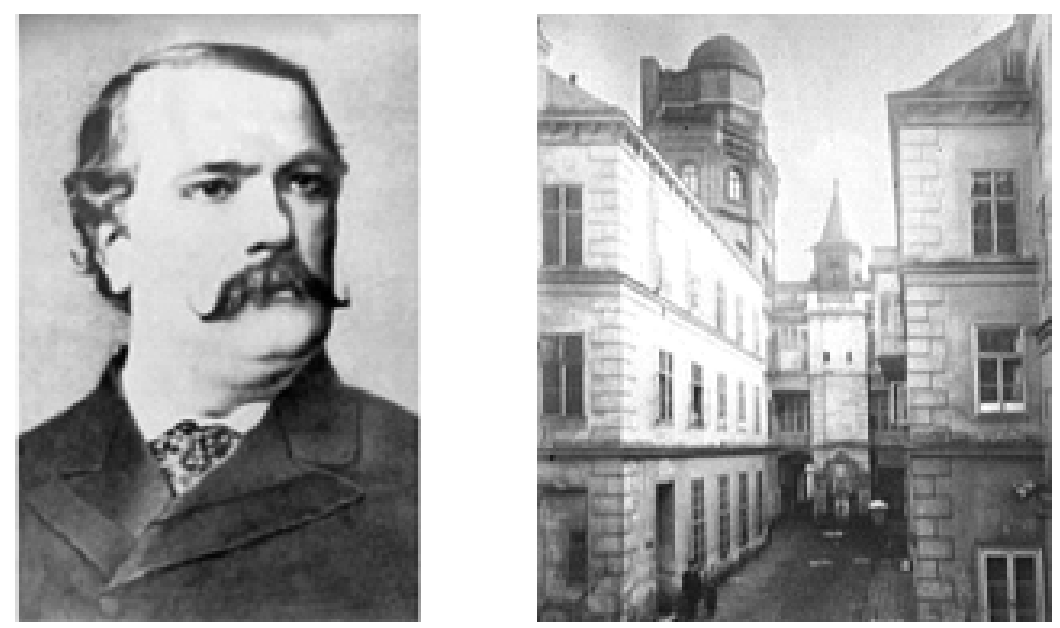

Abb. 1: Links: Theodor v. Oppolzer / Rechts: Sternwarte Wien-Josefstadt

Beobachtungen war ein parallaktisch aufgestelltes 6" - Instrument vorhanden, die Aufstellung erfolgte durch den Werkmeister des Polytechnischen Institutes Chr. Starke. Die damals verwendete Methode zur Bahnbestimmung war die von Gauss, die langwierige und umfangreiche Rechenarbeiten erforderte. Die Arbeiten wurden erst durch eine neue von Theodor von Oppolzer 1870 (Oppolzer 1870) veröffentlichte Methode vereinfacht. Littrow beschäftigt sich ab etwa 1853 mit Arbeiten über die gegenseitige Annäherung dieser Objekte, kommt aber nach etwa 20 Jahren zu dem Schluss, dass diese für eine Massenbestimmung zu selten auftreten.

Theodor von Oppolzer (1841 - 1886) war der Sohn eines berühmten Wiener Arztes und schloss 1865 ebenfalls ein Medizinstudium ab. Sein Interesse galt einzig der Astronomie. Sein Vater richtete ihm im Haus Alserstraße 25 eine Privatsternwarte ein. An dieser Sternwarte Wien-Josefstadt, die als Hauptinstrument einen 7"'-Refraktor hatte (die Optik war ein Dialyt von Plössl, damals wohl das lichtstärkste Objektiv in der gesamten Österreichisch-Ungarischen Monarchie), beobachtete er zwar Kleine Planeten, deren Positionen in den Astronomischen Nachrichten veröffentlicht wurden, sein Hauptinteresse galt aber der Bahnbestimmung und Ephemeridenrechnung von Kometen und Kleinen Planeten. Nach seinen Berechnungen wurden z. B. die verloren gegangenen Objekte (62) Erato, (73) Klytia und (91) Aegina wieder gefunden (z.B. Oppolzer 1865). Oppolzer hatte niemals Astronomie studiert, zur Zeit des Abschlusses des Medizinstudiums hatte er schon 56 astronomische Arbeiten veröffentlicht, 


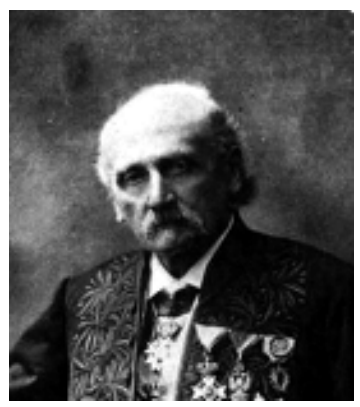

Abb. 2: Moritz Loewy

die für seine Habilitation verwendet wurden. 1870 wurde er außerordentlicher Professor für Astronomie und Höhere Geodäsie an der Universität Wien (Weiss 1887). Oppolzer war mit Moritz Loewy (1833 - 1907) befreundet, der die Wahl seines Lebensweges stark beeinflusste. Loewy hatte in Wien studiert, bekam aber wegen seines jüdischen Glaubens keine Stelle in Wien und ging 1860 nach Paris, wo er sowohl an der Sternwarte als auch am Bureau des Longitudes arbeitete und Vizedirektor sowie Direktor der Sternwarte wurde. Auch Loewy beschäftigte sich ursprünglich mit Asteroiden (Puiseux 1907, Weiss 1887).

1866 begann Johann Palisa (1848 - 1925) in Wien mit dem Astronomiestudium und wurde 1870 an der alten Wiener Sternwarte angestellt. Nach einer kurzen Tätigkeit in Genf bewarb er sich auf Anraten Oppolzers um die Leitung der Marine-Sternwarte in Pola, die er 1872 bekam. Die Aufgaben für Astronomen an der Marinesternwarte waren genau festgelegt: sie hatten Zeitbestimmungen durchzuführen, für die im Hafen liegenden Schiffe das Mittagszeichen abzugeben, die Chronometer der Kriegsmarine regelmäßig zu vergleichen und funktionstüchtig zu erhalten und astronomische, meteorologische und erdmagnetische Beobachtungen anzustellen, soweit es die vorhandenen Hilfsmittel gestatten (J. Palisa 1881). Angehörige des Militärs mit wohlklingenden Titeln wie Linienschiffs-Fähnrich oder Fregattenkapitän, die während ihrer Studienzeit für einige Monate der Wiener Sternwarte für eine Spezialausbildung zum Dienst zugeteilt gewesen waren, arbeiteten mit bescheidenen astronomischen Instrumenten. Palisa bemühte sich, etwas astronomisch Sinnvolles zu tun und begann mit der Beobachtung von und Suche nach Kleinen Planeten. Er legte eigene Sternkarten an, in die er alle Sterne einer bestimmten Himmelsgegend einzeichnete, die mit dem Refraktor in Pola (6 Zoll Öffnung) sichtbar waren. So konnte er die sich relativ zu den Fixsternen bewegenden Asteroiden verhältnismäßig einfach auffinden. Jedes Objekt beobachtete er oft genug, dass eine sichere Bahnbestimmung vorgenommen werden konnte. Am 

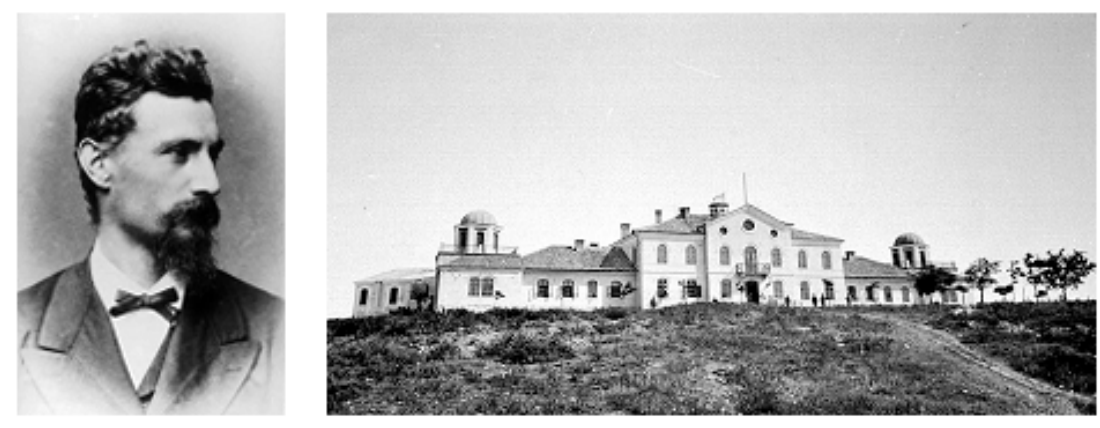

Abb. 3: Links: Johann Palisa / Rechts: Marinesternwarte Pola

18. März 1874 entdeckte er den ersten neuen Kleinen Planeten, das Objekt (136) Austria. Bis zu seinem Weggang von Pola 1880 fand er insgesamt 28. Er beobachtete auch Kometen und bestimmte mit dem Meridiankreis Positionen von Anhaltsternen.

Ein Jahr nachdem Palisa in Pola die Austria entdeckt hatte, gelang auch an der alten Wiener Sternwarte die Entdeckung eines Asteroiden. Leopold Schulhof $(1847$ - 1921) fand am 10. Juli 1875 noch auf der alten Wiener Sternwarte (147) Protogeneia, der Name stammt von Littrow. Schulhof war ebenfalls Jude mit keiner Aussicht auf eine Anstellung in Wien, er ging an das Bureau des Longitudes nach Paris und beschäftigte sich dort vor allem mit Bahnbestimmungen (Bigourdan 1921, 1922).

Palisa reizte natürlich das Angebot von Edmund Weiss, mit dem lichtstarken Großen Refraktor an der neuen Sternwarte in Wien die Beobachtung von Kleinen Planeten unter wesentlich besseren Bedingungen als in Pola durchzuführen. In Wien entdeckte Palisa noch 93 Asteroiden, obwohl er sich von 1892 an hauptsächlich mit der Beobachtung der Objekte befasste, die Max Wolf in Heidelberg auf photographischem Weg gefunden hatte. In Zusammenarbeit mit Wolf entstanden die "Palisa-Wolf-Karten", ein Sternatlas längs der Ekliptik, dessen Herausgabe nach dem Ersten Weltkrieg aus Geldmangel eingestellt werden musste. Die mit einem Gradnetz versehenen Karten zeigten alle Objekte bis zur 15. Größe, insgesamt sind 200 Blätter erschienen. Dies ist ein früher photographischer Himmelsatlas. Mit dem Bruce Teleskop in Heidelberg gewonnene Himmelsaufnahmen wurden in Wien mit einem Gradnetz versehen und dann die Karten gedruckt (Rheden 1945).

Palisa war auch der erste, der sich den neuen Ideen der Astrophysik gegenüber aufgeschlossen zeigte und vor allem die Methoden der Photographie für seine Arbeiten nutzen wollte. Nach einer Untersuchung von Rudolf 

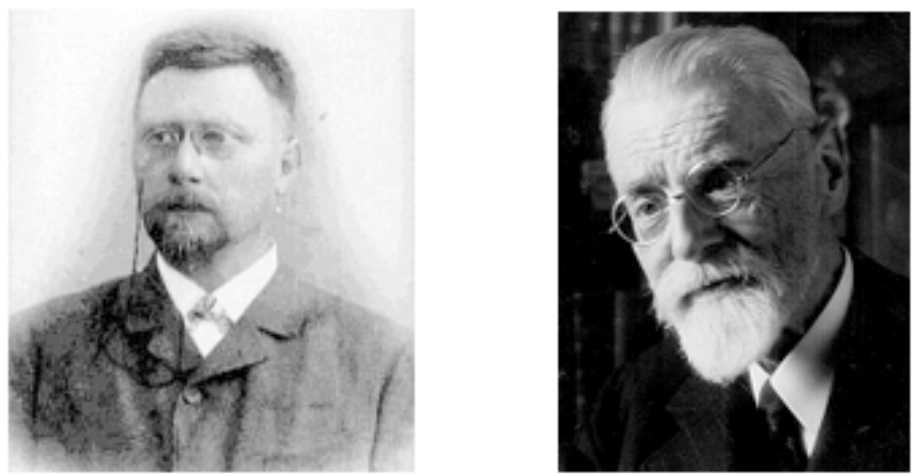

Abb. 4: Links: Rudolf Spitaler / Rechts: Josef Rheden

Spitaler war der Grosse Refraktor mit seinem für den visuellen Spektralbereich korrigierten Objektiv für die damals verwendeten photographischen Emulsionen, deren stärkste Empfindlichkeit im blauen Spektralbereich lag, nur bedingt geeignet (Spitaler 1891).

1885 hatte Palisa Leuten, die einen finanziellen Beitrag für eine von ihm geplante Teilnahme an einer Sonnenfinsternisexpedition leisten würden, die Möglichkeit der Benennung eines Kleinen Planeten versprochen. So fand er auch private Mäzene. Einer von diesen, Freiherr Albert von Rothschild, schenkte der Sternwarte einen Stereokomparator und ein Fernrohr ungewöhnlichen Typs, das Aequatorial Coudé, dessen Konstruktionplan von Moritz Loewy stammt.

1882 nahm Palisa an einer französischen Sonnenfinsternisexpedition zu einer Inselgruppe im Pazifischen Ozean teil. Während der Dauer der Totalität suchte er nach einem damals vermuteten intramerkuriellen Planeten. In populären Vorträgen berichtete er aus seinem reichhaltigen Wissen im Verein zur Verbreitung naturwissenschaftlicher Kenntnisse. Als nach der Pensionierung von Edmund Weiß 1909 wieder ein Theoretiker, Josef Hepperger (1855 - 1928), zum Direktor der Sternwarte ernannt wurde, stellte Palisa aus Protest für einige Zeit seine Beobachtungstätigkeit ein, sicher eine für damalige Verhältnisse mutige Geste. Eine Art Vermächtnis stellt seine letzte Veröffentlichung dar, in der er sich 1924 vehement für die Verlegung der Wiener Sternwarte, die in der Zwischenzeit ja mitten in der Großstadt lag, an einen Ort mit günstigen Beobachtungsbedingungen einsetzte (Palisa J. 1924, Rheden 1925, Hepperger 1925, Oppenheim und Rheden 1925). Palisa erhielt für seine Arbeiten eine Reihe von Preisen, seine sorgfältige Arbeit wurde überall gewürdigt. Fast jedes von ihm entdeckte Objekt wurde nummeriert und benannt, und konnte bei der nächsten Opposition wieder beobachtet werden. Allein (719) Albert war ver- 
loren und wurde erst im Frühling 2000 mit dem Spacewatch Programm wieder gefunden (Larsen, J., McMillan, R.S., Scotti, J.V., Hicks, M., Fevig, R.G., Williams, G.V. 2000). Auch nach seiner Pensionierung 1919 erhielt er das ausdrückliche Recht, seine Beobachtungstätigkeit fortzusetzen.

Friedrich Bidschof (1864 - 1915) und Josef Rheden (1873 - 1946) waren mit Töchtern von Palisa verheiratet. Beide arbeiteten zumindest zeitweise auf dem Gebiet der Asteroiden. Rheden richtete 1908 das Photolaboratorium beim Normalastrographen ein und gewann zahlreiche photogaphische Aufnahmen von Asteroiden.

Etwas ist noch aus der Zeit vor dem Bau der neuen Sternwarte nachzutragen: der spätere Direktor der Sternwarte, Edmund Weiß, bemühte sich anläßlich seiner Reise nach Amerika 1872, um eine Möglichkeit der besseren Verständigung über die Neuentdeckung von Asteroiden zwischen den einzelnen Kontinenten. Er verhandelte mit Christian Heinrich F. Peters, Astronom (Kleinplanetenentdecker) in Clinton. Die transatlantische Kabelgesellschaft bewilligte daher die Übermittlung von Entdeckungsinformationen, die seit 1869 mit der k.u.k. Akademie der Wissenschaften (Oppolzer) ausgetauscht wurden, in beschränkter Anzahl gebührenfrei durchzuführen. Nun wurde zumindest teilweise eine kostenfreie Beförderung innerhalb Europas und Amerikas erwirkt (Littrow, J.J. 1886). Erst 1879 beschäftigte sich die Astronomische Gesellschaft mit diesem Problem (Schönfeld, Winnecke 1879).

Selbstverständlich haben auch alle anderen in Wien angestellten Astronomen Positionen von Asteroiden bestimmt bzw. Bahnbestimmungsrechnungen durchgeführt, zu nennen sind Franz Schaub, August Murmann, Karl Zelbr und Samuel Oppenheim, der, bevor er Professor an der Universität Wien wurde, an der Kuffner Sternwarte und in Prag angestellt war.

Auch in den letzten Jahrzehnten gibt es vereinzelte Arbeiten über Asteroiden. So war die erste wissenschaftliche Arbeit von Alois Purgathofer in Wien 1954 die Bestimmung von 165 Positionen von Nemausa während der Opposition 1954 (Purgathofer 1955). Im Rahmen einer Dissertation wurden manche der mit dem Astrographen aufgenommenen Platten neu vermessen (Pendl 1969) und Lichtkurven von Asteroiden wurden von Hermann Haupt untersucht. Thomas Widorn entwickelte eine Methode zur Bestimmung von Albedo und Durchmesser durch einen Infrarotindex (Widorn 1974). Es beschäftigt sich auch manche der himmelsmechanischen Arbeiten von Rudolf Dvorak und Elke Lohinger mit Asteroiden.

\section{Literatur}

Bigourdan, G. 1921, Anzeige des Todes von Leopold Schulhof, Astron. Nachr. 214, 405

Bigourdan, G.1922, Leopold Schulhof, Bull. Soc. Astr. de France 36, 84 
Firneis,M.G., 1987, Leben und Wirken d. Th.v. Oppolzer, Wien, Geodät. Arbeiten Österr. f.d. internat Erdmessung, N.F.V

Firneis, M.G., Haupt, H., Holl, P.: Sternwarten in Österreich, http://www.austriaca.at/sternwarten

Hepperger, J. 1925, Johann Palisa, Astron. Nachr. 225, 125

Larsen, J., McMillan, R.S., Scotti, J.V., Hicks, M., Fevig, R., G. Williams, G.V. 2000. Discovery and Confirmation of Asteroid (719) Albert $=2000$ JW8. IAU Circ. 7420.

Littrow, J.J. 1835, Die Wunder des Himmels, Hoffmannsche Verlagsbuchhandlung Stuttgart, in 3 Bänden, Band 2, p. 114ff.

Littrow J.J. 1886, Die Wunder des Himmels, 7. Auflage, nach den neuesten Fortschritten der Wissenschaft bearbeitet von Dr.

E. Weiß, Berlin, p. 453

Oppenheim, S., Rheden, J. 1925, Johann Palisa. VJS Astron. Ges. 60, 187

Oppolzer, Th. 1865, Wiederauffindung der Clytia (73), Astron. Nachr. 63, 95

Oppolzer, Th. 1870 bzw. 1880, Lehrbuch zur Bahnbestimmung der Planeten und Kometen. 2 Bände, Leipzig

Palisa, J. 1881, Jahresbericht Pola, VJS Astron. Ges. 16, 227

Palisa J. 1924, Die Verlegung der Wiener Sternwarte eine Notwendigkeit, Wien, Selbstverlag des Verfassers

Pendl, E. 1969, Die Neuvermessung von 140 Aufnahmen Kleiner Planeten sowie ein Programm zur Berechnung vorläufiger Bahnen. Diss. Univ. Wien

Puiseux, P. 1907, Anzeige des Todes von Maurice Loewy, Astron. Nachr. 176, 265

Purgathofer, A. 1955, 165 Positionen des kleinen Planeten (51) Nemausa während der Opposition 1954. Mitt. Univ. Stwte. Wien 7, 235

Rheden, J. 1925, Johann Palisa. Eine kurze Lebensschilderung, Wien, Selbstverlag des Verfassers

Rheden, J. 1945, Die "Palisa-Wolf-Karte", Geschichte eines astronomischen Kartenwerkes, Manuskript, Wien

Schönfeld, E., Winnecke, A.: Bericht über die achte allgemeine Versammlung der Astronomischen Gesellschaft in Berlin, Bericht über die dritte Sitzung, VJS Astron. Ges. 14, p. 345, p. 355,

Spitaler, R. 1891, Zeichnungen und Photographien am Grubbschen Refraktor in den Jahren 1885 bis 1890. Ann.Univ.Stwte.Wien XXIII, 1

Weiss, E. 1887, Nekrolog über Theodor von Oppolzer. Astron. Nachr. 116, 95

Weiss, E. 1908, Maurice (Moritz) Loewy, VJS Astron. Ges. 43, 142

Widorn, Th. 1974 Die Bestimmung der Albedo und des Durchmessers von Asteroiden durch einen Infrarotindex, Ann. Univ.Stwte. Wien, 31, 1 Bildquellen:

Abb. 1a: Institut für Astronomie der Universität Wien

Abb. 1b: Firneis, M.G., Haupt, H., Holl, P.: Sternwarten in Österreich, http://www.austriaca.at/sternwarten

Abb. 2: Weiss, E. 1908, Maurice (Moritz) Loewy, VJS Astron. Ges. 43, 142

Abb. 3a: Österreichisches Staatsarchiv

Abb. 3b: Institut für Astronomie der Universität Wien

Abb. 4a: Institut für Astronomie der Universität Wien

Abb. 4b: Institut für Astronomie der Universität Wien 